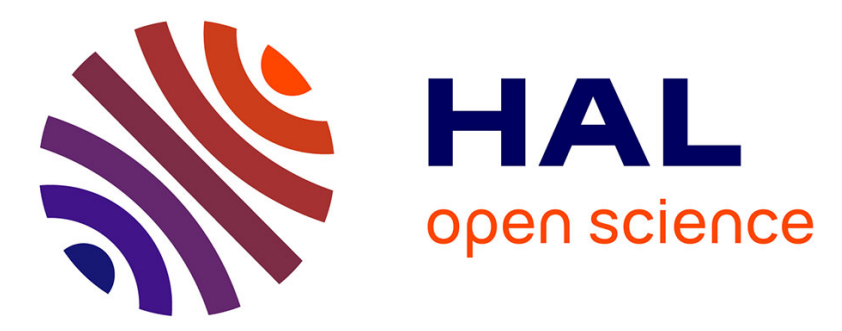

\title{
Orbital Angular Momentum beams generation from 61 channels Coherent Beam Combining femtosecond Digital Laser
}

Matthieu Veinhard, Severine Bellanger, Louis Daniault, Ihsan Fsaifes, Jerome Bourderionnet, Christian Larat, Eric Lallier, Arnaud Brignon, Jean-Christophe Chanteloup

\section{To cite this version:}

Matthieu Veinhard, Severine Bellanger, Louis Daniault, Ihsan Fsaifes, Jerome Bourderionnet, et al.. Orbital Angular Momentum beams generation from 61 channels Coherent Beam Combining femtosecond Digital Laser. Optics Letters, In press, 10.1364/OL.405975 . hal-03038240v2

\section{HAL Id: hal-03038240 \\ https://hal.science/hal-03038240v2}

Submitted on 12 Jan 2021

HAL is a multi-disciplinary open access archive for the deposit and dissemination of scientific research documents, whether they are published or not. The documents may come from teaching and research institutions in France or abroad, or from public or private research centers.
L'archive ouverte pluridisciplinaire HAL, est destinée au dépôt et à la diffusion de documents scientifiques de niveau recherche, publiés ou non, émanant des établissements d'enseignement et de recherche français ou étrangers, des laboratoires publics ou privés. 


\title{
Orbital Angular Momentum beams generation from a 61 channels Coherent Beam Combining femtosecond Digital Laser
}

\author{
Matthieu Veinhard ${ }^{1}{ }^{*}$, Severine Bellanger ${ }^{1}$, Louis Daniault ${ }^{2}$, IhSAn Fsaifes ${ }^{1}$, Jerome \\ Bourderionnet ${ }^{3}$, Christian Larat ${ }^{3}$, Eric Lallier ${ }^{3}$, Arnaud Brignon ${ }^{3}$, And Jean-Christophe \\ Chanteloup ${ }^{1}$ \\ ${ }^{1}$ LULI, CNRS, Ecole Polytechnique, CEA, Sorbonne Université, Institut Polytechnique de Paris, 91120 Palaiseau, France \\ ${ }^{2}$ LOA, ENSTA, Institut Polytechnique de Paris, 91120 Palaiseau, France \\ ${ }^{3}$ Thales Research \& Technology' 1 Avenue Augustin Fresnel, 91767 Palaiseau Cedex, France \\ *matthieu.veinhard@polytechnique.edu
}

Received XX Month XXXX; revised XX Month, XXXX; accepted XX Month XXXX; posted XX Month XXXX (Doc. ID XXXXX); published XX Month XXXX

We report on the use of a 61 beamlets Coherent Beam Combination femtosecond fiber amplifiers as a digital laser source to generate high power Orbital Angular Momentum beams. Such approach opens the path for higher order non-symmetrical user-defined far field distributions. (C) 2020 Optical Society of America

Application fields of Orbital Angular Momentum (OAM) laser beams keep extending, ranging from selective trapping of particles [1], microbunching instability reduction in free-electron laser setups [2] to fluids flow characterization [3] . Traditional methods rely generally on laser cavity tuning [4], phase plates [5], axicons [6] and Spatial-Light Modulators (SLMs) $[7,8]$. The first ones allow high output powers regime operation but lacks tunability, as a different cavity design or optical element must be engineered for each specific transverse beam distribution. The last method offers high tunability but is limited in output powers (peak and average) by the SLM optical damage thresholds. A more versatile alternative approach, offering both high throughput power and tunability, is based on Coherent Beam Combination (CBC) of a large number of beamlines in the far field [9], introducing the concept of a digital laser.

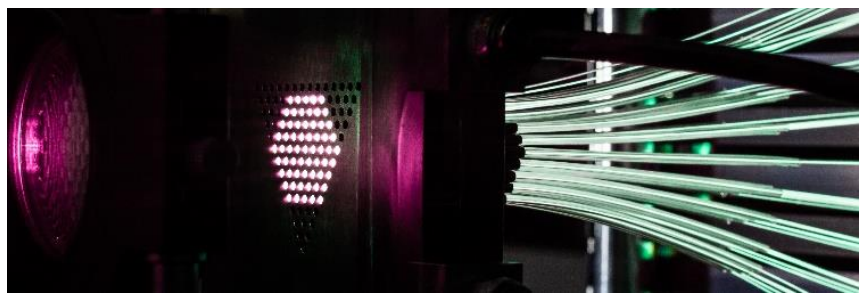

Fig. 1. XCAN laser head (center), collimating microlens array (left) and $61 \mathrm{Yb}$ doped fiber bundle (right). More details about the experimental setup of the $C B C$ of 61 fiber amplifiers are given in reference [10].

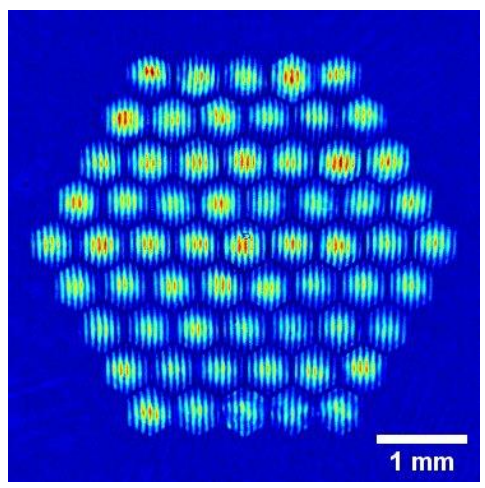

Fig. 2. Near field interference pattern of 61 beamlets as operated on XCAN prototype. Adding successive "pixels" rings will quickly increase the digital laser "resolution".

In this paper, we demonstrate the real-time generation of high power OAM beams using the XCAN CBC laser prototype [10] developed by Ecole Polytechnique and the Thales company.

XCAN architecture relies on the so-called tiled-aperture configuration where $61 \mathrm{Yb}$-fiber amplified laser beams centered at $\lambda=1032 \mathrm{~nm}$ are distributed in the near field side by side as close as possible to each other in a planar hexagonal array arrangement as illustrated on Fig. 1. The figure displays also (left) a hexagonal lenslet aiming at simultaneously collimating all beams. The CBC is then simply achieved when focusing this composite large pupil with a single lens. The electric field transverse distribution observed in this lens focal plane indeed results from the 


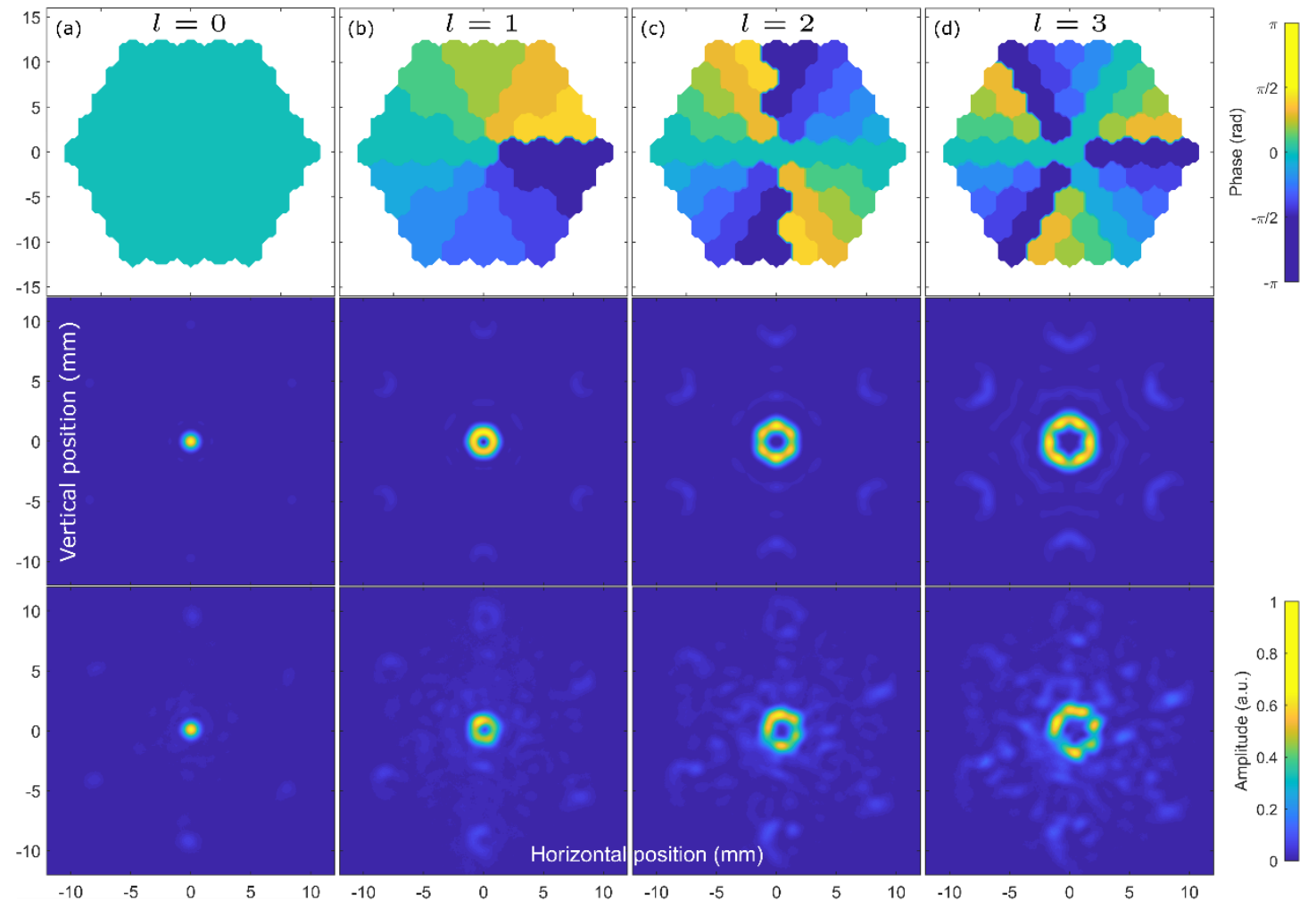

Fig. 3. (top) Computed XCAN phase distributions for $l=0$ (a), 1 (b), 2 (c) and 3 (d) (middle \& bottom) Simulated (middle) and experimental (bottom, $10 \mathrm{~W}$ average power level) far fields for different azimuthal indices $l=0(a), 1(b), 2(c)$ and 3 (d). The reader is invited to play Visualization 1 to appreciate real time switching performances.

coherent addition of all 61 tiled beams accurately phased together. When compared to filled-aperture [11], tiled-aperture CBC is inherently limited in efficiency to the power concentrated into the main lobe in the far field (67\% theoretically) but offers an unrivaled agility in terms of far field beam shaping.

Such key property would indeed be a rather speculative perspective for filled-aperture CBC laser systems where all near field subpupils are superimposed onto each other. We experimentally illustrate here that an XCAN type of laser shall be considered as a femtosecond digital laser where the near field electric field amplitude and phase could be controlled in real time at what one would call the pixel (a single beam) level. The technological choices made while integrating the laser were driven while making sure to keep its high scalability potential. This is especially true at the final power fiber amplifier level where flexiblefiber solutions have been favored. For instance, any rod-type [12] fiber solution was ruled-out to preserve compactness, low cost and scalability.

The phase of each individual beam is controlled in real time $(\mathrm{kHz})$ through the combined use of Variable Optical Delay Lines (VODL) and piezo-mechanical Fiber Stretchers (FS) [10]. The Digital-to-Analog Converter (DAC) addressing the FS currently limits the accuracy at about $\pm \lambda / 50$. This value does not compete with state of the art SLMs $(\lambda / 10000)$ [13] but is more than sufficient regarding CBC requirements [14]. A couple orders of magnitude improvement could nevertheless be achieved by adjusting DAC current voltage accuracy from current $\pm 10 \mathrm{mV}$ down to sub $\mathrm{mV}$ precision while taking adequate electromagnetic environment noise isolation. The phase stabilization is performed using an interferometric measurement method with active phase control and a Stochastic Parallel Gradient Descent (SPGD) algorithm implemented on a Field-Programmable Gate Array (FPGA). A homemade LabVIEW user interface operates the entire experimentand allows to generate a custom set of phases. Each beam amplitude (i.e. power) can be adjusted through amplifier gain control. Fig. 2 displays the near field interference patterns of 61 fibers, illustrating the digital laser concept.

To begin, the laser is operated at $55 \mathrm{MHz}$ repetition rate and low power amplification regime with a total power in the far field around $10 \mathrm{~W}$. Our femtosecond digital laser is then used to generate 61 "pixels" $l$ th-order OAM beam phases expressed as: $\phi(x, y)=l \tan ^{-1}\left[x-x_{0} / y-y_{0}\right]$, where $l \in \mathbb{Z}$ is the azimuthal index (or topological charge), $(x, y)$ a beamlet position relative to the central beamlet position $\left(x_{0}, y_{0}\right)$. No intensity modulation is applied throughout the composite pupil, i.e. each 61 channel carries the same energy. Fig. 3, top images display the 61 fibers set phase distributions allowing to generate $l=0,1,2$ and 3 . The corresponding far fields are displayed in the middle images of Fig. 3 where they are compared to recorded experimental results (bottom images). A rather good agreement between both simulated and experimental far fields for each azimuthal indices can be observed. It is worth noting that the periodic intensity distribution of the composite near field (Fig. 2) gives rise to six side-replica of equal low intensity surrounding a high intensity main beam as observed on the far fields shown in Fig. 3. The same behavior applies when generating donut like shapes. Even more interesting is the fine structure of the rings: lobes equally separated by radial dark stripes. It reveals the azimuthal index value through lobes number: the ring energy appears indeed distributed respectively over 3,2 and 1 lobes when $l=3,2$ and 1 , offering then a simple direct identification of the OAM beam topological charge. Although not displayed here, this feature is numerically confirmed for larger values of $l$ and shall be related to the diffractive 

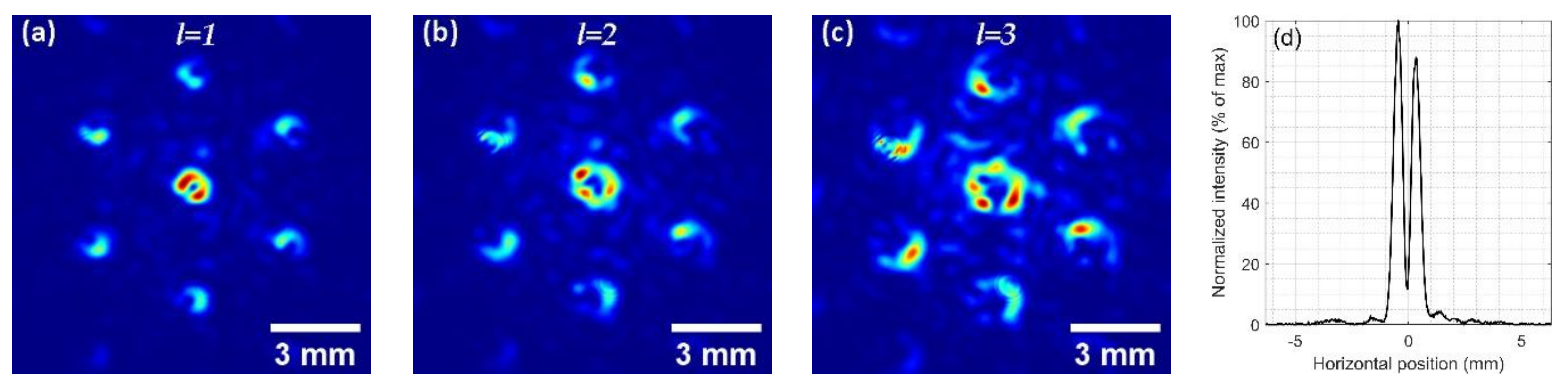

Fig. 4. (a) to (c) Experimental far fields for different azimuthal indices $I=1$ (b) $l=2$ (c) and $I=3$ (d) at high average power regime. (d) Horizontal lineout of $(a)$.

nature of our OAM beam near field pupil [15]. Ongoing work also clearly indicates that a decreased intensity level applied to the peripheral ring of beamlets (therefore attenuating diffraction behavior) greatly improves the ring energy homogenization through dark stripes attenuation.

The video to be played in Visualization 1 first shows the time-varying speckle pattern when the control loop is open; in a second phase, this erratic time varying pattern turns into a stable far field pattern with phase profiles of $l=0,1,2$ and 3 when the control loop is enabled. This experiment demonstrates the digital agility inherent to XCAN 61 pixels architecture where patterns can be switched in real time.

By inserting a pinhole of $3 \mathrm{~mm}$, the far field is then spatially filtered. We measure a far field combining efficiency of $46 \%$ for $l=0$ (As stated above, the theoretical CBC efficiency is $67 \%$ in this case) and of $40 \%$ for $l=1$. Numerical simulations showed that the CBC efficiency for $l=1$ with a $3 \mathrm{~mm}$ pinhole is of $65 \%$. Several approaches have been proposed to qualify annular beams [16] quality. Considering the ratio of above mentioned experimental over theoretical efficiencies leads to Quality Ratios $Q_{R}$ of $69 \%$ and $62 \%$ for $l=0$ and $l=1$. Fiber misalignments in the laser head have been identified as the main source of mechanical improvement to be implemented in the future to near the theoretical values [14].

The laser is then operated in the kW average power regime. As we can observe on Fig. 4, the donut intensity distribution for $l=1$ to 3 azimuthal indices is conserved (although with discontinuities in the ring) up to $740 \mathrm{~W}$ of total power (including main and side lobes powers).

When ramping the laser up to $1 \mathrm{~kW}$ average power, we observed severe degradation of the donut pattern, mainly related to thermal effects within the laser head. However, we are confident that a thermal management upgrade of the laser will allow the generation of clean $\mathrm{kW}$ average power OAM beams with far field qualities close to low operation regime (Fig. 3).

XCAN CBC amplifying stage is integrated into a CPA architecture in order to deliver sub-ps pulse trains. Thus, prior to power amplification and CBC operation, the pulses are temporally stretched to $2.5 \mathrm{~ns}$ through a Chirped Fiber Bragg Grating (C-FBG). The combined and spatially filtered beam is ultimately temporally compressed by using a $1750 \mathrm{l} / \mathrm{mm}$ multilayer dielectric reflection grating pair.

The compressed pulse autocorrelation of the donut-shape beam is depicted in Fig. 5. Gaussian fit gives a FWHM duration of $533 \mathrm{fs}$, corresponding to a $378 \mathrm{fs}$ pulse duration assuming a Fourier Transform limited pulse. As shown on the insert of Fig. 5, the donut intensity distribution is rather well conserved after compression, illustrating the compatibility of CPA architecture with OAM beams generation and preservation [17]. Although out of the scope of this work, more detailed characterization of femtosecond pulses carrying orbital angular momentum shall ultimately be performed [18].
Our experimental setup has been implemented and studied within an up to 10,000 channels compatibility context [19], therefore paving the way to very high-resolution transverse amplitude and phase digital laser beam control. Hundreds to thousands of channels, each being a control point within the pupil, will indeed allow arbitrary shaped phase and amplitude distributions (not limited to cylindrical symmetries like

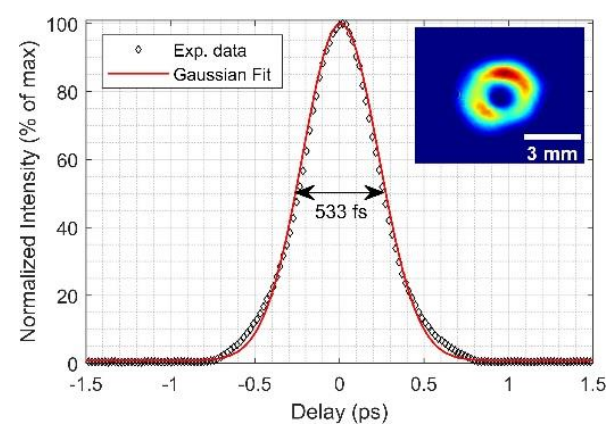

Fig. 5. Autocorrelation trace for an OAM beam of $l=1$. Gaussian fit gives a FWHM duration of $533 \mathrm{fs}$ leading to $378 \mathrm{fs}$ pulse duration. Inserted image: $I=1$ far field profile after compression.

for OAM) to be implemented. This can be performed for instance with iterative electric field computing approaches like Gerchberg-Saxton (GS) type of algorithm [20]. The GS algorithm computes the required phase to be applied on the near field to produce a target amplitude in the far field. Each iteration relies on a 2D Fourier transform applied on the near field, followed by an inverse Fourier transform applied on the resulting phase, previously combined with the target amplitude.

Numerical simulations were therefore performed with the implementation of a GS algorithm. We started by defining the composite initial near field, with the desired number of channels. Each channel initial phase was randomly chosen within $[-\pi, \pi]$. Two simulations of different far field targets were performed. In the first one, the target was defined as a square supergaussian beam, as shown in the top image of Fig. $6 \mathrm{~b}$ while, for the second run, the target was defined as the Ecole Polytechnique logo $(\mathrm{X})$, as shown in the bottom image of Fig. 6b. For both runs, results were obtained with a near field composed of 9919 beamlets in a hexagonal arrangement ( 57 rings around the central fiber vs. only 4 for the XCAN laser). Those computed phases are shown in Fig. 6a (top image for the square target and bottom image for the $\mathrm{X}$ logo) while the resulting far fields are displayed in Fig. 6c. It can clearly be seen that with a sufficient number of beamlets, it is feasible to achieve userdefined intensity distributions in the far field.

In summary, we have demonstrated that theXCAN laser and its highly scalable architecture shall be considered as the advent of a new laser 

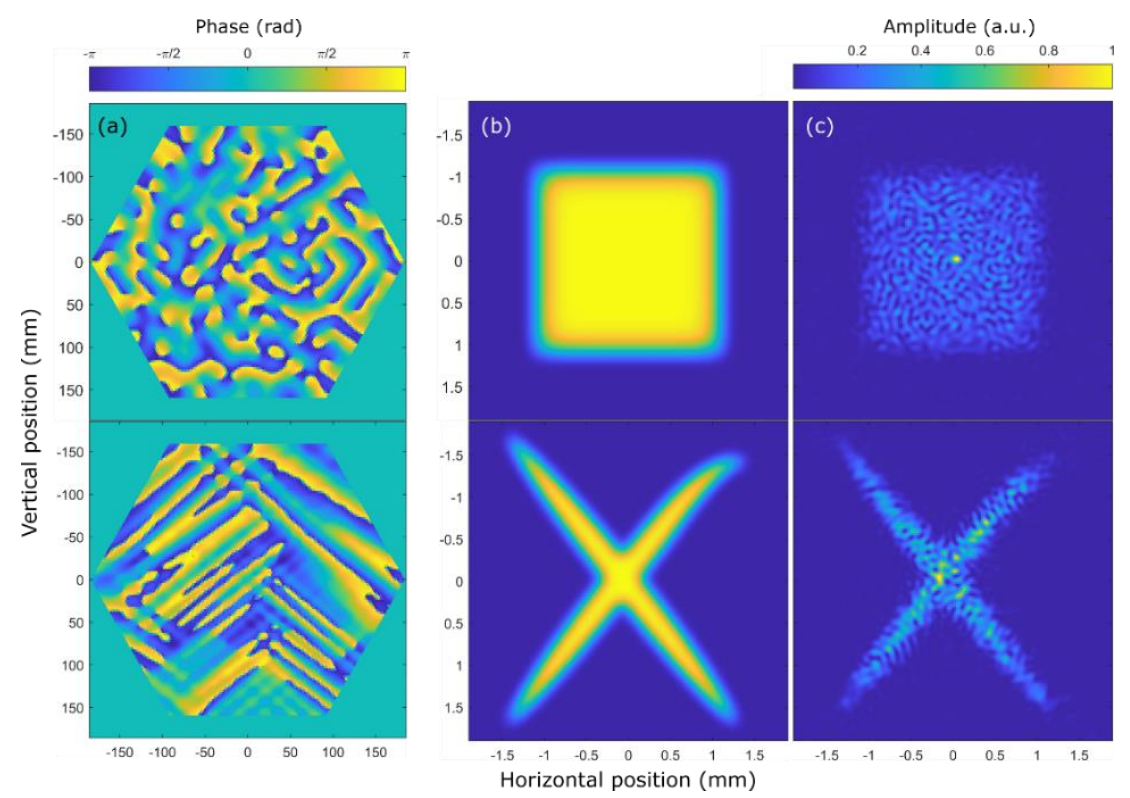

Fig. 6. (a) Composite phases calculated by the GS algorithm (Top image for a square supergaussian target and bottom image for the Ecole Polytechnique logo). (b) Target far fields. (c) Computed final far fields.

architecture paradigm: the digital laser. Such approach carries a high potential for greatly broadening the field of applications of structured light far beyond OAM centrosymmetric beams only. It indeed associates high peak (fs regime operation) and average (kW regime) powers thanks to its diode-pumped Yb-doped fiber amplifying chain architecture. Compact and scalable by nature, it has been conceived and implemented (with a 1280x1024 resolution camera) to be compatible with up to 10,000 channels paving the way to high-resolution transverse amplitude and phase laser beam control. It has indeed been demonstrated that as low as 8 camera pixels per beamlet would be sufficient to ensure an efficient CBC [19]. It is also worth noting that XCAN carries as well longitudinal pulse energy distribution tailoring ability thanks to its pulse shaper [10] and, although not implemented yet on our experimental setup, individual polarization adjustment could also be foreseen.

Finally, these beam properties real time adjustability (currently up to the $\mathrm{kHz}$ regime) are offering an extra degree of freedom for applications (drilling, cutting or soldering of material for instance) requesting dynamic energy or power distribution control.

\section{Funding}

This project has received funding from the French Ministry of Defense (Agence de l'Innovation de Défense) and the European Union's Horizon 2020 research and innovation programme under grant agreement no 654148 Laserlab-Europe.

\section{Disclosures}

The authors declare no conflicts of interest.

\section{References}

1. G. Tkachenko and E. Brasselet, Nat. Commun., vol. 5, 2014

2. N. Liebster, J. Tang, D. Ratner, W. Liu, S. Vetter, Z. Huang, and S. Carbajo, Phys. Rev. Accel. Beams, vol. 21, no. 9, 2018

3. A. Ryabtsev, S. Pouya, A. Safaripour, M. Koochesfahani, and M. Dantus, Opt. Express, vol. 24, no. 11, 2016

4. A. Mock, D. Sounas, and A. Alù, Phys. Rev. Lett., vol. 121, no.10, 2018
5. C. Dorrer, Opt. Lett., vol. 34, no. 15, 2009

6. N. Ochiai, J. Shou, and Y. Ozeki, J. Opt. Soc. Am. B, vol. 36, no. 5,2019

7. L. Zhu and J. Wang, Sci. Rep., vol. 4, no. 1, 2014

8. S. Ngcobo, I. Litvin, L. Burger, and A. Forbes, Nat. Commun., vol. 4, no. 1, 2013

9. R. Lemons, W. Liu, C. G. Durfee, J. C. Frisch, S. Smith, J. Robinson, A. Fry, and S. Carbajo, in Conference on Lasers and Electro-Optics, 2019

10. I. Fsaifes, L. Daniault, S. Bellanger, M. Veinhard, J. Bourderionnet, C. Larat, E. Lallier, E. Durand, A. Brignon, and J.-C. Chanteloup, Opt. Express, vol. 28, no. 14, 2020

11. M. Müller, C. Aleshire, A. Klenke, E. Haddad, F. Légaré, A. Tünnermann, and J. Limpert, Opt. Lett., vol. 45, no. 11, 2020

12. J. Limpert, N. Deguil-Robin, I. Manek-Hönninger, F. Salin, F. Röser, A. Liem, T. Schreiber, S. Nolte, H. Zellmer, A. Tünnermann, J. Broeng, A. Petersson, and C. Jakobsen, Opt. Express, vol. 13, no. 4, 2005

13. https://www.meadowlark.com/ (accessed Nov. 17, 2020)

14. A. Heilmann, J. Le Dortz, L. Daniault, I. Fsaifes, S. Bellanger, J. Bourderionnet, C. Larat, E. Lallier, M. Antier, E. Durand, C. Simon-Boisson, A. Brignon, and J.-C. Chanteloup, Opt. Express, vol. 26, no. 24, 2018

15. Y. Liu and J. Pu, Opt. Commun., vol. 284, no. 10-11, 2011

16. J. B. Ohland, U. Eisenbarth, M. Roth, and V. Bagnoud, Appl. Phys. B Lasers Opt., vol. 125, no. 11, 2019

17. Y. C. Lin, Y. Nabekawa, and K. Midorikawa, Appl. Phys. $B$ Lasers Opt., vol. 122, no. 11, 2016

18. M. Miranda, M. Kotur, P. Rudawski, C. Guo, A. Harth, A. L'Huillier, and C. L. Arnold, J. Mod. Opt., vol. 64, no. 4, 2017

19. M. Antier, J. Bourderionnet, C. Larat, E. Lallier, E. Lenormand, J. Primot, and A. Brignon, IEEE J. Sel. Top. Quantum Electron., vol. 20, no. 5, 2014

20. R. W. Gerchberg and W. O. Saxton, Optik (Stuttg)., vol. 35, 1972 